\title{
Angina bullosa hemorrhagica, an uncommon oral disorder. Report of 4 cases
}

\author{
Javier Alberdi-Navarro ${ }^{1}$, Abel García-García ${ }^{2}$, Francisco Cardona-Tortajada ${ }^{3}$, María-Luisa Gainza-Cirauqui ${ }^{4}$, \\ José-Manuel Aguirre-Urizar ${ }^{1}$
}

\begin{abstract}
${ }^{1}$ Oral Medicine and Oral and Maxillofacial Pathology Units. Dental Clinic Service, Department of Stomatology II, School of Medicine and Nursing, University of the Basque Country (UPV/EHU), Leioa, Bizkaia, Spain

${ }^{2}$ Oral Medicine, Oral Surgery and Implantology Unit, Faculty of Medicine and Dentistry, University of Santiago de Compostela, Health Research Institute of Santiago de Compostela (IDIS), Santiago de Compostela, A Coruña, Spain

${ }^{3}$ Oral Health Department, Navarre Health Service-Osasunbidea, Pamplona, Spain

${ }^{4}$ Department of Dental Surgery, Faculty of Dental Surgery, University of Malta, Msida, Malta
\end{abstract}

Correspondence:

Oral Medicine and Oral and Maxillofacial Pathology Units

Dental Clinic Service, Department of Stomatology II

School of Medicine and Nursing

University of the Basque Country (UPV/EHU)

48940 Leioa, Bizkaia, Spain

alberdinavarrojavier@gmail.com

\begin{abstract}
Alberdi-Navarro J, García-García A, Cardona-Tortajada F, Gainza-Cirauqui ML, Aguirre-Urizar JM. Angina bullosa hemorrhagica, an uncommon oral disorder. Report of 4 cases. J Clin Exp Dent. 2020;12(5):e509-13.
\end{abstract}

Received: 21/01/2020

Accepted: 24/02/2020

\begin{abstract}
Angina bullosa hemorrhagica (ABH) is a rare oral disorder characterized by blood-filled bullous lesions in the oral cavity and the oropharynx in the absence of an underlying systemic, haematological or mucocutaneous condition. The presentation of the lesions is acute and located on the lining mucosa, mainly on the soft palate. Often, these lesions are single and rupture easily leaving an ulcerated area. In this study, we present $4 \mathrm{ABH}$ cases in 3 women and 1 man and we discuss the main clinicopathological characteristics. The characteristics of this disorder are important to recognize in order to differentiate the lesions from other oral bullous conditions of the oral cavity such as mucocutaneous disorders or blood coagulation disorders.
\end{abstract}

Key words: Angina bullosa hemorrhagica, angina bullosa haemorrhagica, oral blisters.

\section{Introduction}

Angina bullosa hemorrhagica $(\mathrm{ABH})$ is a rare benign condition characterised by subepithelial blood-filled bullae in the oral or oropharyngeal mucosa, in absence of dermatological, haematological or systemic disorders (1).

The real prevalence and incidence of $\mathrm{ABH}$ are unknown although it is considered to be present in $0.05 \%$ of patients seen in specialized units of Oral Medicine and
Oral Pathology $(2,3)$. Generally, this disorder is seen in adults over 30 years of age, with a peak incidence in the 5 th decade of life and without a clear gender predilection (1).

The etiopathogenesis of $\mathrm{ABH}$ is yet unknown and therefore considered multifactorial. Trauma on the oral mucosa is considered to precipitate the typical bullous lesions in susceptible individuals (4).

This disorder is characterized clinically by the sudden 
onset of one or more red-blue blood-filled subepithelial bullae, mainly on the soft palate, lateral borders of the tongue and buccal mucosa (4-6). The time of evolution of the bullae is variable but generally short as they rupture leaving a superficial ulcer that heals within one or two weeks without scarring $(1,5,7,8)$. Recurrent lesions are frequent, appearing on the same or in another location $(2,6)$.

In the rare cases where a biopsy was taken, the histopathological features of $\mathrm{ABH}$ were non-specific, presenting as a blood-filled subepithelial bulla with atrophic epithelium and chronic inflammatory infiltrate or as a non-specific superficial ulceration, covered by a fibrinopurulent membrane and chronic inflammatory infiltrate $(2,4,9)$.

Diagnostic criteria for $\mathrm{ABH}$ have been suggested recently (Table 1) (6).

The management for this disorder is symptomatic and focuses on healing of the ulcers and in preventing the onset of new lesions. The overall prognosis of $\mathrm{ABH}$ is good, although there are cases where obstruction of the airway has been reported $(1,10)$.

In this paper, we analyze the main clinicopathological characteristics of 4 cases diagnosed as ABH by applying the proposed criteria for this condition and by performing a differential diagnosis with the main blood-filled vesiculo-bullous conditions of the oral cavity.

\section{Case Report}

Case 1: A 28-year-old male, with no systemic conditions, toxic habits or known allergies, was seen at the clinic presenting with multiple blood-filled vesiculo-bullous lesions on the right soft palate (Fig. 1A). These lesions were asymptomatic and would appear each time the patient would take a plane, having two previous episodes with similar lesions that healed ad integrum in 2-3 days. There were no other lesions on the skin or other mucosae, no general abnormalities, and the results of the blood tests, including coagulation profile, were normal. Diagnostic criteria of $7 / 9$.

Case 2: A 22-year-old woman presenting to consultation with blood-filled blistering lesions on the soft palate and the uvula of a sudden onset (Fig. 1B). The patient does not refer any other intraoral or extraoral abnormalities. This manifestation has been present twice, with no correlating factors. The bullae last between 1 and 2 days causing discomfort when swallowing and speaking. Once the bullae rupture, the ulcerated lesions healed in a few days without scarring. The results of the blood test, including coagulation profile, were non-significant. Diagnostic criteria of 6/9.

Case 3: A 62-year-old woman presenting with sudden and recurrent blood-filled blisters on the lateral border of the tongue (Fig. 1C). The patient presented with a 10year history of surgical removal of thyroid cancer currently in treatment with levothyroxine sodium (100 mg/ day). The blisters were asymptomatic and ruptured after 2-4 hours, while the succeeding ulcer healed within 7 days without scarring. The patient did not present with any other cutaneous, mucosal or systemic lesions. The results of the blood tests, including coagulation profile, were non-significant. Diagnostic criteria of 8/9.

Case 4: A 49-year-old woman, with no relevant medical history, presenting for the last months with recurrent blood-filled blisters on the soft palate, lateral border of the tongue and buccal mucosa. These lesions ruptured in 3-6 hours leaving an ulcerated surface (Fig. 1D) that caused slight discomfort and healed without scarring in 7 days. Other cutaneous or mucosal lesions were not noticed and the results of the blood test, including coagulation profile, were non-significant. Diagnostic criteria of $7 / 9$.

The main clinical characteristics of the 4 cases are presented in Table 2.

Table 1: Clinical criteria for the diagnosis of ABH as proposed by Ordoini et al., 2019 (6).

\begin{tabular}{|c|c|}
\hline I & $\begin{array}{c}\text { Clinically notable haemorrhagic bulla or erosion with a history of } \\
\text { bleeding of the oral mucosa }\end{array}$ \\
\hline II & Exclusively oral or oropharyngeal localization \\
\hline III & Palate localization \\
\hline IV & Triggering event or food promoting factor (food intake) \\
\hline V & Recurrent lesions \\
\hline VI & Favourable evolution without a scar within few days \\
\hline VII & Painless lesion, tingling or burning sensation \\
\hline VIII & Normal platelet count and coagulation profile \\
\hline IX & Negative direct immunofluorescence \\
\hline
\end{tabular}

For a positive diagnosis of $\mathrm{ABH}$ using these criteria, the case should meet a minimum of 6 out of 9 defined criteria, with criteria I and II as required (6). 


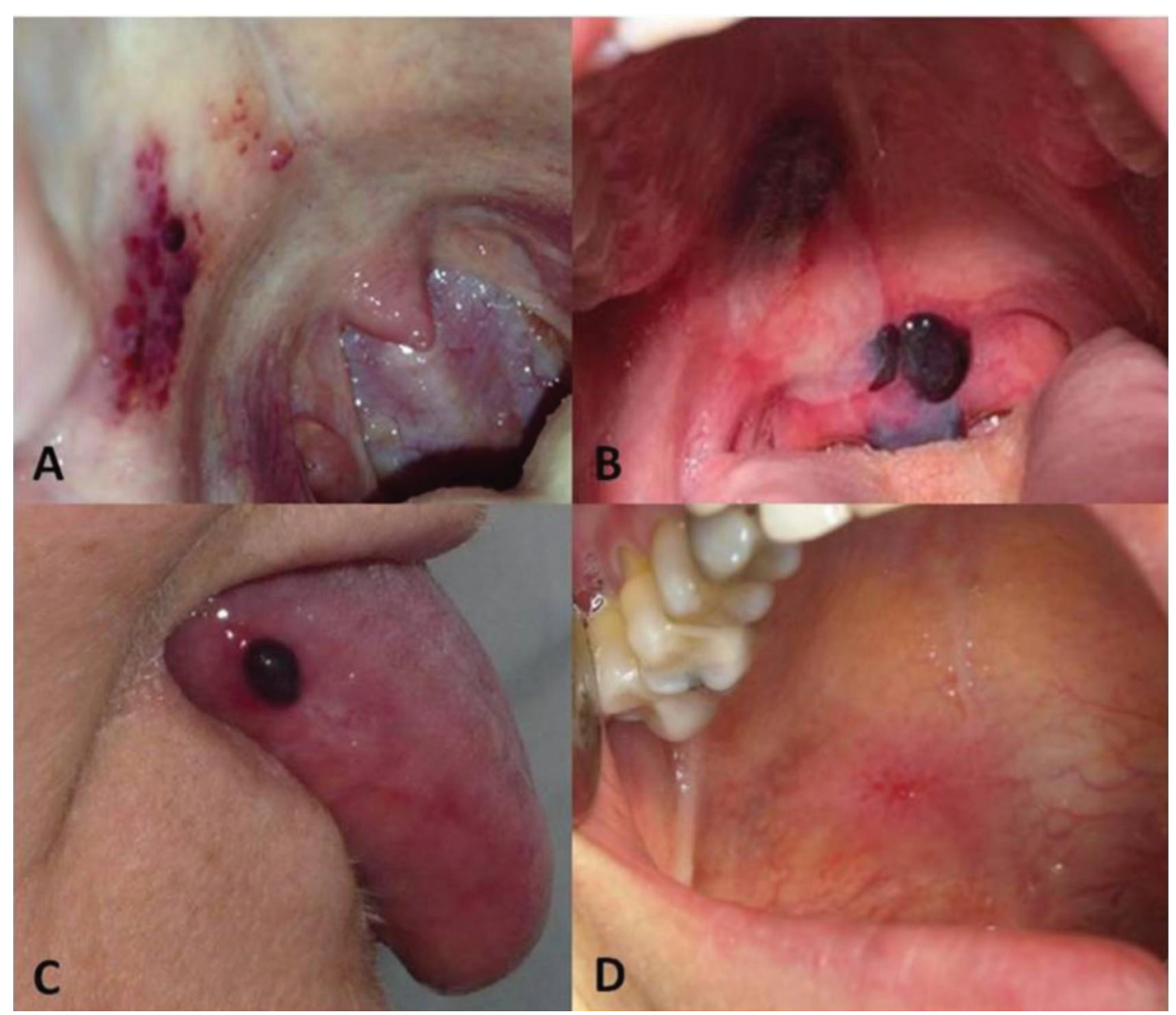

Fig. 1: A) Blood-filled vesiculo-bullous lesions on the soft palate. Case 1. B) Bullous lesions on the soft palate and uvula, showing some of the lesions rupture. Case 2. C) Blood-filled bullous lesion on the lateral border of the tongue in case 3. D) Ulcerated area following rupture of the bullous lesion in case 4.

Table 2: Data of the cases presented in this study.

\begin{tabular}{|c|c|c|c|c|c|c|c|c|}
\hline Case & Age/Sex & Cause & Location & $\begin{array}{l}\text { Number } \\
\text { of lesions/ } \\
\text { flare-up }\end{array}$ & $\begin{array}{c}\text { Time of } \\
\text { evolution }\end{array}$ & Symptoms & Recurrence & $\begin{array}{c}\text { Number of } \\
\text { DC Ordioni } \\
\text { et al. } 2019 \\
\text { (6). }\end{array}$ \\
\hline 1 & $28 / \mathrm{M}$ & Flight & Soft palate & $4-6$ & 2-3 days & No & 2 episodes & $7 / 9$ \\
\hline 2 & $22 / \mathrm{F}$ & NR & Soft palate & $2-3$ & 1-2 days & Soreness & 2 episodes & $6 / 9$ \\
\hline 3 & $62 / \mathrm{F}$ & NR & $\begin{array}{l}\text { Soft palate, lateral } \\
\text { border of the tongue, } \\
\text { buccal mucosa }\end{array}$ & 1 & 2-4 hours & No & 4 episodes & $8 / 9$ \\
\hline 4 & $49 / F$ & NR & $\begin{array}{l}\text { Soft palate, lateral } \\
\text { border of the tongue, } \\
\text { buccal mucosa }\end{array}$ & 1 & 3-6 hours & Soreness & $\begin{array}{l}\text { Multiple epi- } \\
\text { sodes }\end{array}$ & $7 / 9$ \\
\hline
\end{tabular}

M: Male; F: Female; NR: No relation; CD: Diagnostic Criteria.

\section{Discussion}

The ABH is an intriguing benign pathological entity of the oral cavity initially defined by Badham in 1967 (11), although it had been previously described with different names such as hemorrhagic bullous stomatitis, localized oral purpura or stomatopompholyx hemorrhagica $(2,9)$. The etiology of this benign oral pathology is still unknown and it is considered to occur in susceptible patients 
following a traumatic event $(1,6)$. Among the described traumatic factors are consuming hard or crunchy food, certain dental treatments or even medical procedures such as an intubation $(1,10,12)$. The onset of $\mathrm{ABH}$ has also been linked to certain drug therapies, mainly topical steroids, as well as, although more controversial, with certain systemic conditions such as hypertension and diabetes mellitus $(1,6)$. The use of topical steroids is known to cause epithelial atrophy and alteration of the elastic fibres of the lamina propria, which may promote the formation of subepithelial bullous lesions following trauma $(13,14)$. In our case, none of the patients were being treated with topical or inhaled steroids. We were only able to identify the traumatic cause in one of the cases (Case 1) where the patient referred the association between presenting the bullae and travelling by airplane. This has been previously recorded and we believe it can be associated with atypical movements and/or with changes in environmental pressure causing oral mucosal trauma and leading to the onset of the bullae.

In 2019, Ordoini et al., (6) published a proposal of diagnostic criteria for $\mathrm{ABH}$ following a systematic review of previously published cases. According to these authors, the diagnosis of $\mathrm{ABH}$ is given if the case fulfills 6 out of 9 of the proposed criteria, with criteria 1 and 2 as required (Table 2). These criteria are clinical in essence and suggest that, although other mucocutaneous and haematological pathologies should be excluded, this should be done only when the clinical presentation of $\mathrm{ABH}$ is atypical (6). All of our cases fulfilled the diagnostic criteria as they all met more than 6 criteria. Although we believe that this proposal for diagnostic criteria is the first approach in defining ABH objectively, we consider that further studies are required to analyze their sensitivity and specificity.

An important aspect to consider in $\mathrm{ABH}$ is the location of the lesions. As described in other studies (4-6,8), the most common site of appearance of the bullous lesions is the soft palate, and therefore is recognized as a diagnostic criteria for $\mathrm{ABH}$ (6). In our case, 3 of the patients presented with lesions in this location. This predominance for the soft palate may be related to a higher risk of trauma during mastication and swallowing, and because of a thin mucosal epithelial lining that may be damaged more than other more protected sites, such as the hard palate and the gingiva.

The differential diagnosis of $\mathrm{ABH}$ must be done with vesiculobullous conditions of the oral mucosa, primarily haematological and mucocutaneous conditions of an immunological origin $(1,5,6)$.

Some haematological disorders that may have a clinical presentation similar to $\mathrm{ABH}$ in the oral cavity are thrombocytopenia, von Willebrand disease, leukaemia and some vasculitis $(9,15)$. In order to exclude these disorders, it is necessary to perform a thorough medi- cal history and examination, as well as a blood analysis that includes the complete blood count and coagulation profile. In our case, all of our patients presented normal blood test results, including coagulation profile.

The main group of conditions included in the differential diagnosis are mucocutaneous diseases of an immunological origin, including various conditions such as mucous membrane pemphigoid, pemphigus vulgaris, lineal IgA disease, acquired epidermolysis bullosa and bullous amyloidosis (1). These conditions are ruled out by examining other mucosae and skin for other lesions.

We observed a high recurrence rate of ABH lesions in our cases since all patients expressed having previous episodes. This rate is higher than the $62 \%$ reported by Ordioni et al., in 2019 (6).

Regarding treatment of this benign condition, we should highlight that currently there is no specific treatment for $\mathrm{ABH}$. Management of the symptoms with topical antiseptics, such as chlorhexidine in concentrations between $0.12 \%$ and $0.2 \%$ has been described (6). In addition, eliminating irritants or traumatic components that may cause recurrence of the lesions is indicated $(2,8)$.

\section{Conclusions}

We may state that $\mathrm{ABH}$ is a rare pathology of the oral mucosa that should be included in the differential diagnosis of blood-filled bullous lesions in the oral cavity. Knowing the characteristics of this oral pathology is key in its diagnosis. The applicability of the proposed diagnostic criteria for $\mathrm{ABH}$ should be assessed through further controlled studies.

\section{References}

1. Alberdi-Navarro J, Gainza-Cirauqui ML, Prieto-Elías M, Aguirre-Urizar JM. Angina bullosa hemorrhagica an enigmatic oral disease. World J Stomatol. 2015;4:1-7.

2. Grinspan D, Aulafia J, Lanfanchi H. Angina bullosa hemorrhagica. Int J Dermatol. 1999;38:525-8.

3. Rosa A, Geraldo Pappen F, Neutzing Gomes AP. Angina bullosa hemorrhagica: a rare condition? RSBO. 2012;9:190-192.

4. Giuliani M, Favia GF, Lajolo C, Miani CM. Angina bullosa haemorrhagica: presentation of eight new cases and a review of the literature Oral Dis. 2002;8:54-8.

5. Dias KB, Flores AP, Oliveira MG, Carrand VD, Hildebrand LC, Sant'Ana Filho M. Angina bullosa hemorrhagica: report of 7 cases and analysis of 199 cases from the literature. Gen Dent. 2017;65:31-36.

6. Ordioni U, Hadj Saïd M, Thiery G, Campana F, Catherine JH, Lan R. Angina bullosa haemorrhagica: a systematic review and proposal for diagnostic criteria. Int J Oral Maxillofac Surg. 2019;49:28-39.

7. Yamamoto K, Fujimoto M, Inoue M, Maeda M, Yamakawa N, Kirita T. Angina bullosa hemorrhagica of the soft palate: report of the 11 cases and literature review. J Oral Maxillofac Surg. 2006;64:1433-6.

8. Horie N, Kawano R, Inaba J, Numa T, Kato T, Nasu D, et al. Angina bullosa of the soft palate: a clinical study of 16 cases. J Oral Sci. 2008; 50: $33-6$

9. Stephenson P, Lamey PJ, Scully C, Prime SS. Angina bullosa haemorrhagica: clinical and laboratory features in 30 patients. Oral Surg Oral Med Parol Pathol. 1987;63:560-565.

10. Pahl C, Yarrow S, Steventon N, Saeed NR, Dyar O. Angina bullosa haemorrhagica presenting as acute upper airway obstruction. Br J Anaesth. 2004;92:283-6. 
11. Badham NJ. Blood bullae and the oesophageal cast. J Laryngol Otol. 1967;81:791-803.

12. Sato Y, Yokoyama K, Watanabe J, Nakamura A. Pharyngeal angina bullosa heamorrhagica due to EGD. Gastrointest Endosc. 2018;87:1583-4.

13. High AS, Main DM. Angina bullosa haemorrhagica: a complication of long-term steroid inhaler use. Br Dent J. 1988;165:176-179.

14. Martini MZ, Lemos CA, Shinohara EH. Angina bullosa hemorrhagica: report of 4 cases. Minerva Stomatol. 2010;59:129-142.

15. Rivera C, Torres Í, González-Arriagada WA. Haemorrhagic bullae in the oral mucosa with gingival bleeding. BMJ. 2017; 359: j4476.

Conflict of interest

Non declared. 